\title{
La Focalis-action : Des savoirs narratifs aux faires vidéoludiques
}

Hugo Montembeault et Bernard Perron

\section{(2) OpenEdition \\ Journals}

Édition électronique

URL : http://journals.openedition.org/sdj/897

DOI : $10.4000 /$ sdj. 897

ISSN : 2269-2657

Éditeur

Laboratoire EXPERICE - Centre de Recherche Interuniversitaire Expérience Ressources Culturelles Education

\section{Référence électronique}

Hugo Montembeault et Bernard Perron, «La Focalis-action : Des savoirs narratifs aux faires vidéoludiques », Sciences du jeu [En ligne], 9 | 2018, mis en ligne le 30 mai 2018, consulté le 19 avril 2019. URL : http://journals.openedition.org/sdj/897 ; DOI : 10.4000/sdj.897

Ce document a été généré automatiquement le 19 avril 2019

Tous droits réservés 


\title{
La Focalis-action : Des savoirs narratifs aux faires vidéoludiques
}

\author{
Hugo Montembeault et Bernard Perron
}

1 À l'occasion d'une remise en perspective des relations entre le narratif et le ludique souhaitée par ce numéro de Sciences du jeu, il apparait incontournable d'aborder et de questionner l'un des concepts phares de la narratologie : la focalisation. Après un survol des théories littéraires et cinématographiques qui ont fondé l'utilisation du concept, nous allons examiner leurs applications en études vidéoludiques afin d'apporter des clarifications et des ajustements conceptuels. Nous souhaitons proposer une compréhension de l'expérience vidéoludique narrative qui tienne compte: 1) des stratégies de design intégrant la transmission de devoir-faire et de pouvoir-faire dans un cadre narratif et 2) de la manière dont le joueur apprend, comprend et s'approprie ces stratégies afin de réguler l'actualisation de ses vouloir-faire et de ses savoir-faire à travers sa jouabilité. Pour mieux aborder ces derniers aspects, nous estimons pertinent de les définir en termes de focalis-action.

\section{Les foyers de la théorie}

2 On se souvient que pour Gérard Genette, qui introduit la notion en études littéraires dans son « Discours du récit » de Figures III (1972), la focalisation s'intègre à la sous-catégorie de la perspective, distinction faite par le narratologue entre le mode (« qui voit?») et la voix (« qui parle?»). C'est dans son Nouveau discours du récit en 1983 que Genette définit plus clairement sa pensée :

Par focalisation, j'entends donc bien une restriction de «champ », c'est-à-dire en fait une sélection de l'information narrative par rapport à ce que la tradition nommait l'omniscience... L'instrument de cette (éventuelle) sélection est un foyer 
situé, c'est-à-dire une sorte de goulot d'information, qui n'en laisse passer que ce qu'autorise sa situation... (Genette, 1983, p. 49).

3 À partir de ce modèle, Genette propose trois types de focalisation (Genette, 1972, p. 206-208) que l'on peut résumer de la sorte :

1. La focalisation zéro (Narrateur > Personnage): le récit dans lequel le narrateur « omniscient » en sait plus et en dit plus que n'en savent aucun des personnages.

2. La focalisation interne (Narrateur $=$ Personnage) $:$ le récit dans lequel le narrateur dit uniquement ce que sait tel ou tel personnage et où tout est filtré par la conscience de celuici.

3. La focalisation externe (Narrateur < Personnage) : le récit dans lequel le narrateur en sait moins que le personnage et où il est impossible pour le premier de pénétrer la conscience du second.

Cette définition et cette typologie ont été reprises et révisées à maintes occasions. Mieke Bal a notamment été l'une des premières à critiquer ce système en études littéraires. Elle insiste sur l'importance de repenser le concept autour d'un "centre d'intérêt» superposant une opinion ou une attitude subjective face aux contenus narratifs sélectionnés, présentés et visualisés (Bal, 1977, p. 119). Chez Bal, voir, c'est aussi concevoir . La focalisation apparaît ainsi comme une opération autonome de mise en discours qui comporte ses propres figures, niveaux et instances. Dans ce modèle, il y a «un focalisateur, qui n'est pas le narrateur [...] mais qui n'est pas non plus le focalisé » (Bal, 1977, p. 119). Le focalisateur peut être situé à l'intérieur (donc interne) ou à l'extérieur de la diégèse (externe). Pour sa part, le focalisé correspond à l'événement, le lieu, l'action, le personnage, l'objet, etc., qui est perçu par un focalisateur. Cette distinction entre le sujet de la vue (le focalisateur) et l'objet de la vue (le focalisé, "perceptible » lorsque présenté de l'extérieur ou « imperceptible » lorsque présenté depuis son intérieur) est au cœur de la contribution de Bal à l'étude de la focalisation.

5 En voulant étudier les images et les sons du septième art, les narratologues ont à leur tour dû apporter d'autres nuances. Pour Jean-Paul Simon (1983), c'est la correspondance entre le point de vue de la caméra et l'identité du foyer narratif (personnage, narrateur ou instance invisible) qui permet de déterminer si la focalisation est interne, externe ou si le récit est non-focalisé. Inspirée de Bal, la pensée de Michèle Lagny, Marie-Claire RoparsWuilleumier et Pierre Sorlin (1984) détermine quant à elle le mode de la focalisation en fonction de l'interdépendance entre la caméra qui voit et les contenus narratifs qui sont vus. Si le travail de la caméra est omniscient, libre de toutes contraintes et sans intentions claires de manipuler l'information visuelle, il s'agit de focalisation zéro. Si l'activité perceptive et l'information narrative apparaissent comme étant délibérément régulées par la caméra elle-même (ses cadrages, ses mouvements, ses angles, ses effets optiques, etc.), la focalisation est nommée interne-caméra. Finalement, le récit devient en focalisation interne si l'articulation de la caméra est reliée à l'activité perceptive d'un personnage.

Dans L'Eil-caméra: entre film et roman, Jost (1987) différencie de son côté trois modes de régulation de l'information narrative. Il conserve le terme «focalisation » pour désigner la manière dont le récit du méga-narrateur (chez Gaudreault) ou grand imagier (chez Laffay) distribue les savoirs narratifs entre le spectateur et les personnages. La grande différence tient à la focalisation zéro qui est vue comme une focalisation spectatorielle puisque le récit donne un avantage cognitif au spectateur qui en sait plus que les personnages. Jost introduit ensuite les termes «ocularisation» pour caractériser la 
relation entre ce que la caméra montre et ce qu'un personnage est censé voir ainsi que " auricularisation " pour le " point de vue » sonore. Ces notions seront qualifiées de zéro lorsque le visuel ou le sonore sont gérés par l'omniscience du grand imagier. Jost parle d'ocularisation/auricularisation: 1) interne primaire du moment que l'image ou le son apparaissent comme étant filtrés par le corps d'un personnage sans avoir recours au contexte formel du film pour trancher sur la question et 2) interne secondaire dès l'instant où l'ancrage de l'image et du son dans la subjectivité d'un personnage dépend d'une contextualisation des procédés formels du montage image et du montage son.

Enfin, et de manière fort intéressante pour notre perspective, André Gardies pense la focalisation en termes de " pouvoir ludique »: « En ce sens, la focalisation, dès lors qu'elle a pour fonction de régler mon savoir diégétique, apparait comme l'un des arguments majeurs d'un échange ludique" (1988, p.144). Cet échange relève selon lui d'une «stratégie de l'énonciation» qui implique une relation de "partenaire-adversaire » (p. 139) entre le spectateur et ce qu'il nomme l'énonciateur ; Gardies fait même directement référence à l'agôn de Roger Caillois pour développer sa réflexion.

Lorsque Bernard Perron s'intéresse à la focalisation, il constate que les précédentes théories sont trop liées à la typologie et à la « transcendance d'une instance locutrice ou narratrice qui conditionne[nt] les travaux de Genette » (Perron, 1991, p. 27). Il remarque que le couplage exclusif entre la focalisation et la narration pose problème. Dans un médium audiovisuel comme le cinéma, la manipulation des savoirs narratifs dépend inévitablement d'une régulation de l'information visuelle, sonore et narrative. Or, il est inutile de démultiplier et de complexifier les typologies en dissociant entre autres localisation et monstration (chez Gardies) ou encore ocularisation, auricularisation et focalisation (chez Jost). Puisque vision, audition et narration sont toujours imbriquées et manipulées dans un film, le cadre de la focalisation est plutôt fixé par des opérations d'attribution spectatorielle qui dépendent de l'acte de «lecture » et de la sagacité du spectateur à être sensible aux procédés de focalisation. Pour remettre en cause la toutepuissance d'un narrateur omniscient ou d'une caméra envisagée comme instance privilégiée de régulation des contenus narratifs, Perron s'inspire de la théorie de l'énonciation impersonnelle de Christian Metz (1991) et du cognitivisme. Il s'éloigne ainsi de l'idée d'une délégation des foyers de perception pour envisager la question à partir de l'instance spectatorielle et de sa compréhension de l'histoire. Dans cette perspective, c'est le spectateur qui attribue le cadre la focalisation.

La pertinence de recourir à la notion d'attribution - et d'effectuer un retour théorique en arrière de 25 ans - prend tout son sens lorsqu'il devient difficile de savoir d'emblée à qui le narrateur aurait délégué la responsabilité d'un sous-récit. Par exemple, dans Mission: Impossible (Brian De Palma, 1996), l'agent Ethan Hunt (Tom Cruise) et son chef de mission Jim Phelps (John Voight) qu'il pensait mort se retrouvent dans un café londonien et discutent de l'opération avortée à Prague, opération ayant suivi le générique d'ouverture. Des retours en arrière audiovisualisent les événements passés - notamment l'un de ceuxlà sous deux angles différents et selon deux versions distinctes - sans qu'il soit possible lors d'un premier visionnement de les imputer de façon irréfutable à Hunt ou à Phelps puisque les retours au présent, eux, alternent entre les deux personnages (Figure 1). 
Figure 1

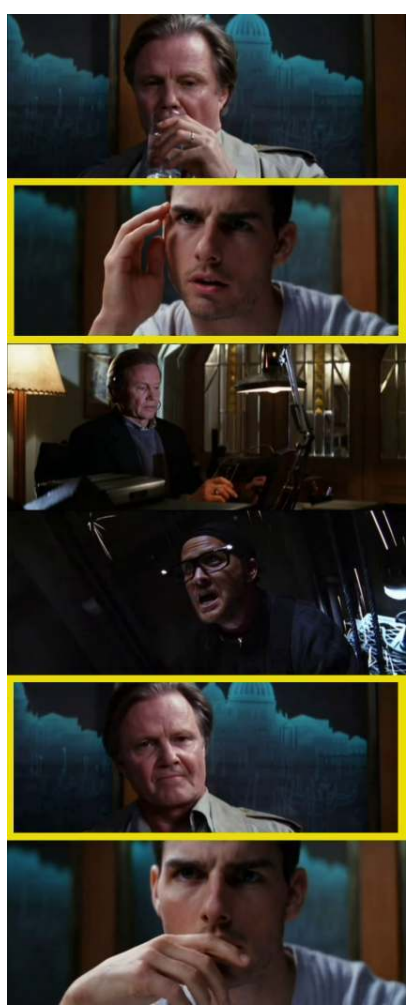

Un retour en arrière dans Mission : Impossible (Brian De Palma, 1996) amorcé par l'agent Ethan Hunt (cadre jaune du haut) et clos dans le temps présent par son chef de mission Jim Phelps (cadre jaune du bas).

La construction narrative de cette confusion spectatorielle au sujet de l'identification du personnage responsable de la sous-narration entraîne des conséquences sur la compréhension de l'histoire et l'interprétation du récit enchâssé. D'une part, si le spectateur attribue ces retours en arrière à Phelps, c'est que celui-ci pense aux circonstances véridiques qu'il est encore en train de taire à son interlocuteur et que par conséquent, il relance sa tromperie. D'autre part, si le spectateur les attribue à Hunt (alors que des images viennent contredire ce qu'il dit à Phelps), cela signifie que ce dernier prend conscience de la supercherie dont il a été la victime et que cette découverte pourra être déterminante quant à ses actions futures. En clair, c'est la manière dont le spectateur assigne le foyer de la perception (Hunt ou Phelps) qui permet d'établir le statut narratif (vérité ou mensonge) des événements remémorés dans cette séquence de Mission: Impossible. C'est pour cette raison que Perron propose de réfléchir l'attribution de ce foyer à partir de diverses configurations qui peuvent renvoyer à trois origines distinctes : soit à une origine implicite que le spectateur attribue à une conscience abstraite (donc une "focalisation »; focalisation sans adjectif ou déterminant puisque tout est toujours focalisé sinon il n'y aurait pas de récit filmique), soit à une origine explicite ou anthropomorphisée hors de la diégèse (le réalisateur ou le configurateur de la focalisation) ou finalement à un personnage dans l'univers du récit (le focalisateur). 


\section{Pour mieux focaliser la théorie narratologique du jeu vidéo}

11 Sans surprise, c'est la typologie genettienne et les propositions de Bal qui dominent l'application du concept de focalisation dans les études du jeu vidéo. Il en résulte un ancrage encore beaucoup trop axé sur les instances narratives subsumant l'ensemble de la sélection, de la présentation et de la vision des contenus vidéoludiques. Nous allons nous limiter à des tenants narratifs pour amorcer notre réflexion avant de poursuivre vers des aboutissants vidéoludiques.

Michael Nitsche, l'un des premiers théoriciens à s'y référer, va utiliser la focalisation dans l'optique de Bal pour analyser les jeux narratifs en 3D. Selon lui, la focalisation en jeu vidéo ne peut pas être qu'une simple «copie» de celle empruntée aux traditions cinématographiques. Puisque la caméra interactive « détachée » donne la possibilité au joueur de diriger son regard où il le souhaite, Nitsche (2005, p. 4) associe une importante part de la focalisation à la configuration audiovisuelle de l'espace. C'est un argument qu'il reprend intégralement dans son livre Video Game Spaces: Image, Play and Structure in 3D Worlds (2008). L'architecture, la disposition d'indices visuels et la mise en cadre de l'espace (cadrage, recadrage, mouvement, effet optique, etc.) influencent ainsi la sélection de l'information narrative parce qu'elles agissent comme des «attracteurs » ou des «opportunités perceptuelles» (Nitsche, 2005, p. 4 et 2008, p. 152) qui aiguillent la manipulation de la caméra virtuelle. ${ }^{1}$

13 Tout en laissant de côté l'idée du focalisé, il différencie les focalisateurs interne et externe. Il donne l'exemple de Super Mario 64 (Nintendo, 1996) et note : «Les joueurs contrôlent à la fois Mario, l'acteur [performer] de toutes les actions pertinentes dans le monde $\mathrm{du}$ jeu, et le focalisateur externe qu'est Lakitu» (Nitsche 2005, p.2). Cette observation soulève des interrogations dans la mesure où le personnage de Lakitu est visualisé à plusieurs reprises dans la diégèse de Super Mario 64 (Figure 2 - image du haut). Plus encore, la petite tortue s'adresse directement à Mario lors du tutoriel portant sur l'utilisation de la «Lakitu Cam ». Or, si l'on joue le jeu des instances narratives, cette caméra fictionnelle que tient Lakitu figure comme focalisateur interne au sens de Bal ; et non pas comme focalisateur externe tel que le dit Nitsche ${ }^{2}$ (Figure 2 - image du bas). 


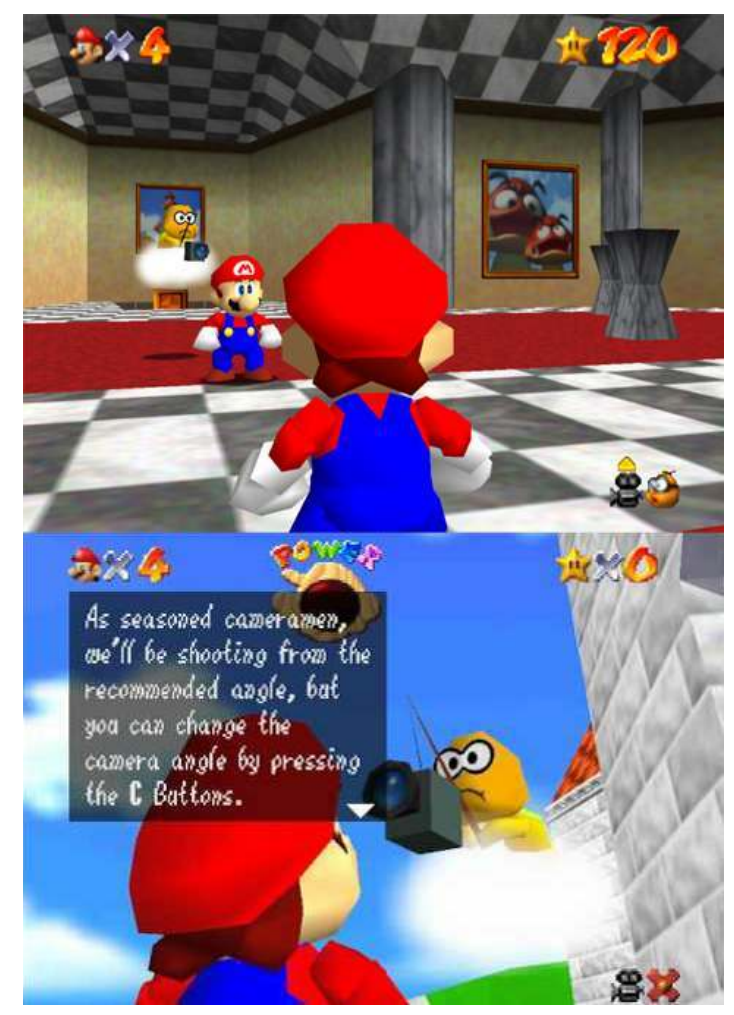

Dans Super Mario 64 (Nintendo, 1996), Lakitu en focalisateur interne.

Suivant les propositions de la narratologue, qu'occulte finalement ce dernier, il est aussi nécessaire d'indiquer que les apparitions de Lakitu à l'écran durant le tutoriel font de ce personnage un focalisé perceptible, dévoilant ici à l'existence d'un autre focalisateur qui est cette fois externe ; autrement, à qui attribuer le point de vue lorsque le joueur voit Lakitu filmer Mario durant le tutoriel ? Par ailleurs, dès l'instant où le foyer de la perception est repositionné dans l'objectif de la «Lakitu Cam » et que le joueur reprend le contrôle de ladite caméra, il peut osciller entre l'attribution de la présentation de l'information à la petite tortue (focalisateur, interne suivant la théorie de Bal), à lui-même comme configurateur de la focalisation contrôlant Lakitu, ou au deux à la fois, entre autres parce que Mario reste visible à l'écran et qu'en incarnant le fameux plombier, le joueur finit par oublier que la caméra virtuelle est diégétisée (comme le fait d'ailleurs Nitsche). Cette dernière observation pointe vers une distinction commune en jeu vidéo qui est claire sur le plan visuel, mais ambivalent sur le plan de l'expérience ; c'est-à-dire celle entre les jeux à la troisième personne et ceux à la première personne.

Suivant la réflexion de Tamer Thabet dans Video Game Narrative and Criticism : Playing the Story: «Le joueur de jeux à la troisième personne n'est pas un personnage-focal [focalcharacter] et ne peut être qu'un focalisateur externe puisque l'illusion visuelle qui le place dans le corps du personnage n'est pas créée » (Thabet, 2015, p. 42). Quant aux jeux à la première personne, le joueur « occupe l'espace et perçoit à travers les yeux et les oreilles du protagoniste; il ou elle fait l'expérience de l'histoire à la place du protagoniste et de manière générale devient le protagoniste » (Thabet, 2015, p. 43). Au premier abord, cette dichotomie a du sens. Elle peut toutefois être revisitée, ce vers quoi pointe une autre 
remarque de Thabet à propos des jeux à la première personne, mais qui reste applicable à l'incarnation d'un personnage dans d'autres régimes de vision :

Contrairement aux formes traditionnelles de fiction, dans lesquelles nous lisons ou regardons le conflit d'un protagoniste et d'un antagoniste et dans lesquelles notre réaction ne se produit que dans nos esprits plus ou moins empathiques, dans la fiction des jeux, le conflit est plus concrètement le nôtre dès lors où nous nous trouvons à l'intérieur du corps du protagoniste. Ainsi, l'histoire devient une expérience personnelle qui nous renseigne [tells] beaucoup plus sur nous-mêmes que sur le protagoniste (Thabet, 2015, p. 43).

Dès l'instant où le joueur a un sentiment de présence à l'intérieur de l'univers du jeu, la distinction entre les focalisateurs externe et interne devient plus floue et brouille au passage le statut même de sujet de la focalisation; et ce, peu importe le régime de la vision. C'est pour cette raison qu'il est courant que se chevauchent différentes relations d'attribution du foyer de la perception. Le sentiment d'être "là » et de se sentir personnellement concerné par les événements est en effet un état ressenti à la première personne, non pas dans le sens narratif et visuel, mais bien dans le sens psychologique. Nous rejoignons ici la théorie cognitiviste d'Edward Branigan.

L'auteur de Narrative Comprehension and Film reconnaît trois principaux types de narration ou d'agentivité (agency): "un narrateur formule des affirmations au sujet de [offers statements about]; un acteur/agent agit sur ou est celui sur lequel on agit; et un focalisateur fait l'expérience de » (Branigan, 1992, p. 105). En ce sens, la focalisation reste « une tentative de représenter la conscience de [quelque chose] » (p. 106) et « se prolonge à l'expérience plus complexe d'objets: penser, se souvenir, interpréter, s'étonner, craindre, croire, désirer, comprendre, se sentir coupable » (p. 101). Pour Branigan, on ne peut pas traiter le focalisateur comme un seul instrument de distribution du savoir narratif ou comme une instance égale à un narrateur ; il faut plutôt le définir comme un "expérimentateur». Afin de traduire la possibilité que le personnage-acteur puisse devenir et être un focalisateur, Branigan (1992, p. 106). fait chevaucher ces deux sphères d'agentivité (l'acteur et le focalisateur) dans le cadre d'une figure ( $\left.n^{\circ} 25\right)$. Adapté au jeu vidéo, on comprend que le joueur se situe précisément à l'intersection de ces deux sphères. Peu importe la perspective visuelle (première ou troisième), le joueur ne voit pas simplement l'univers du jeu par l'entremise de son personnage-joueur, visible ou non, il s'y conçoit comme « centre d'intérêt » et comme expérimentateur. Ne serait-ce que par la seule exploration de l'espace vidéoludique fictionnel, le joueur se transporte par la force de l'imagination dans un autre monde et se retrouve au cœur de l'expérience des événements. Par exemple, dans un jeu vidéo d'épouvante à la troisième personne, plus que le personnage-joueur, c'est souvent davantage lui qui a peur ou qui est terrorisé. Dans ces conditions, en tant qu'expérimentateur du jeu, le joueur est fréquemment amené à se considérer lui-même, et non pas son personnage, comme focalisateur dont la sélection de l'information dépend entièrement de ses compétences perceptivo-cognitives et sensorimotrices. À cet instant, son personnage-joueur est envisagé comme un focalisé perceptible ou imperceptible (dans le cas des jeux à la première personne). ${ }^{3}$ Évidemment, les cinématiques changent la donne. En retirant le contrôle au joueur, elles repositionnent ce dernier en tant que spectateur, lui rappelant l'existence de configurations de la focalisation mises en place par le système du jeu ou par le concepteur de jeu s'il peut anthropomorphiser ce dernier. 

sont posées par le travail de Gordon Calleja qui s'y réfère de manière très générale lorsqu'il développe son concept d'alterbiographie qui renvoie à

la construction active d'une histoire en cours qui se développe grâce à l'interaction avec la topographie du monde du jeu, les habitants, les objets, les règles de jeu et la physique qui est codée. Les alterbiographies sont générées par le joueur qui adopte une attitude narrative à l'égard de l'interprétation de certains signes représentationnels et des opérations mécaniques qui les animent (Calleja, 2009, p. 5). focalisation comme une « restriction de champ » adopté par le joueur et qui détermine l'interprétation narrative des propriétés formelles du jeu. La contribution de l'auteur à l'étude de la focalisation réside dans le fait que ce travail interprétatif dépend du sujet alterbiographique que le joueur situe comme "centre d'intérêt ». Pour Calleja, il existe trois sujets alterbiographiques: les miniatures (plusieurs personnages localisables à différents endroits dans le monde du jeu comme dans un jeu de stratégie en temps réel), l'entité (que contrôle le joueur dans des jeux à la première et la troisième personne) et le soi (c'est-à-dire le joueur lui-même) (Calleja 2011, p. 125-126). L'actualisation d'une alterbiographie dépend alors du choix de focalisateur. Dans le cas du jeu de stratégie en temps réel par exemple, si le joueur décide d'actualiser l'alterbiographie des unités de combat sur le champ de bataille (miniatures), il produira un récit tout autre que s'il adopte le point de vue d'un général de guerre en surplomb (entité) ou encore son propre point de vue de joueur (soi). Le brouillard de la guerre (fog of war) peut alors être lu comme le résultat d'une topographie n'ayant pas été explorée soit par les miniatures, soit par le joueur lui-même, ou même par l'entité quand il s'agit d'un commandant lors d'une campagne de Starcraft (Blizzard, 1988). Suivant cette perspective, la construction d'un bâtiment, qui peut prendre des heures en temps fictionnel contrairement à quelques secondes en temps de jouabilité, pointe vers cette multiplicité des récits alterbiographiques selon l'agent de la focalisation sélectionné pour interpréter les événements ludo-narratifs et les interactions de jeu.

L'avancée conceptuelle de Calleja demeure malheureusement encore négligée par les théoriciens de la focalisation en jeu vidéo. L'application somme toute problématique de la focalisation genettienne en études vidéoludiques qui est effectuée par Fraser Allison permet de réaffirmer l'importance de l'expérience et de l'attribution dans la transposition théorique. Mirror's Edge (EA DICE, 2008), une hybridation entre le jeu de plateforme et le jeu d'action-aventure qui se joue en perspective à la première personne, constitue l'objet d'étude principal de l'auteur dans "Whose mind is the signal? Focalization in video game narratives " (Allison, 2015). L'histoire prend place dans un environnement urbain contemporain et le joueur incarne Faith, une messagère professionnelle dont le travail consiste à livrer des informations secrètes et des colis illégaux. Allison s'attarde entre autres au travail de la musique. Au cours des moments où Faith se fait remarquer par les policiers, des fusillades ainsi que des courses-poursuites ont lieu. Ces situations sont toujours accompagnées d'une musique extradiégétique très rythmée et haute en décibels. Représentant selon Allison (2015, p.11) «la montée d'adrénaline » chez Faith, le déclenchement de la musique " peut être considéré comme un élément de focalisation interne [car] il communique ou met l'accent sur l'état émotionnel $[\mathrm{du}]$ personnage ». Or, jusqu'à preuve du contraire, puisque Faith n'entend pas cette musique extradiégétique, il semble étrange de l'évaluer dans l'optique de la 
focalisation, même interne (puisqu'il s'agit davantage d'une question d'énonciation). Or, si l'on joue le jeu du raisonnement narratologique et que l'on reprend le modèle de Jost pour continuer à transposer les modèles cinématographiques, on aurait plutôt affaire à une focalisation "spectatorielle» (le joueur en sait plus que le personnage) en ocularisation interne primaire (voyant par l'entremise des yeux du personnage) et en auricularisation zéro (par l'écoute d'une musique "hors de la diégèse » non filtrée à travers la subjectivité du personnage). Ce n'est donc pas l'expérience de Faith qui est davantage dramatisée, mais bien celle de celui qui joue. Durant ces séquences interactives d'action, le joueur s'attribue plus explicitement le rôle de focalisateur et l'alterbiographie est davantage la sienne.

21 Ailleurs dans son article, Allison s'intéresse au système de guidage spatio-narratif nommé le "Sens urbain ", un mode de visualisation qui applique une texture rouge foncé sur les objets de l'environnement de jeu nécessaires à la navigation optimale. Cette mécanique de jeu est en fait, suivant Genette, "une sorte de goulot d'information ». Dans la perspective d'Allison, ce "Sens urbain» est attribué à l'attention que Faith porte aux éléments importants de l'espace en tant qu'experte de la pratique du parcours; il serait question de focalisation interne. L'auteur poursuit son raisonnement en indiquant que la possibilité de désactiver le "Sens urbain » (c'est-à-dire de retirer le surlignage en rouge du parcours idéal) entraîne un renversement : « [Désactiver le "Sens urbain"] augmente la difficulté pour le joueur, mais cela fait également basculer la focalisation de la vision du personnage expert vers une focalisation externe plus neutre, car le joueur est obligé de remplacer les motifs mentaux du personnage par les siens » (Allison 2015, p. 10) (Figure 3).

Figure 3

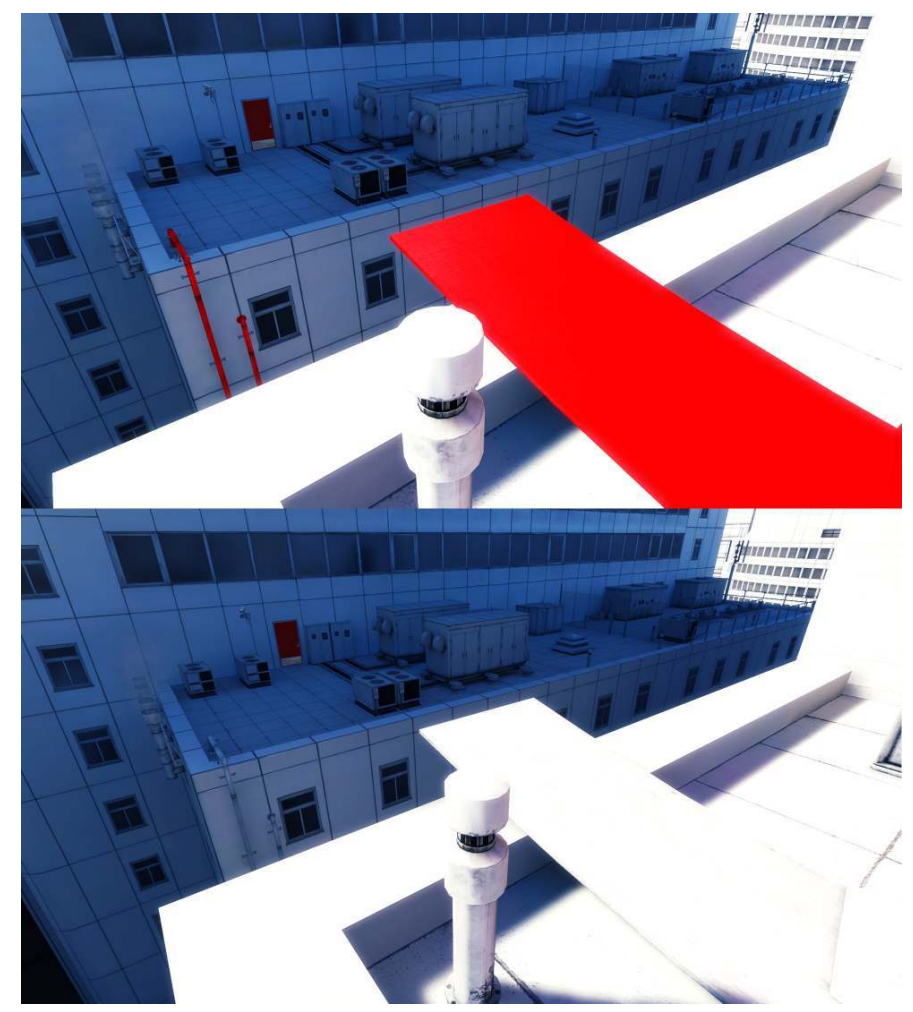

Images de Mirror's Edge (EA DICE, 2008) avec le « Sens urbain » activé (en haut) et désactivé (en bas) 
Or, pour montrer à nouveau le flottement d'une approche genettienne basée sur la délégation du foyer de la perception par une instance narrative, on constate qu'il est tout aussi plausible de relier l'activation du « Sens urbain » à la focalisation zéro (le narrateur en montre plus que n'en voit/sait réellement Faith) et sa désactivation à la focalisation interne (le narrateur montre à travers les yeux experts de Faith). Ce faisant, relier le «Sens urbain» de Mirror's Edge à la focalisation interne et son retrait à la focalisation externe comme le fait Allison n'est recevable que si le joueur impute à Faith le rôle de focalisateur au seul personnage. Si le joueur attribue le tracé rouge au processus abstrait de la focalisation ou encore au travail des concepteurs de jeu, ce code de couleur apparaîtra comme une marque du système de jeu ou comme une configuration de la focalisation. Enfin, s'il s'impute à lui-même la décision d'activer ou de désactiver cette signalisation colorimétrique, il peut se considérer comme le configurateur de la focalisation. D'ailleurs, rien dans le tutoriel ne dit que c'est Faith qui voit les éléments en rouge; c'est bien le joueur qui est interpelé et sa vision qui est dirigée vers lesdites opportunités interactives (Figure 4).

Figure 4

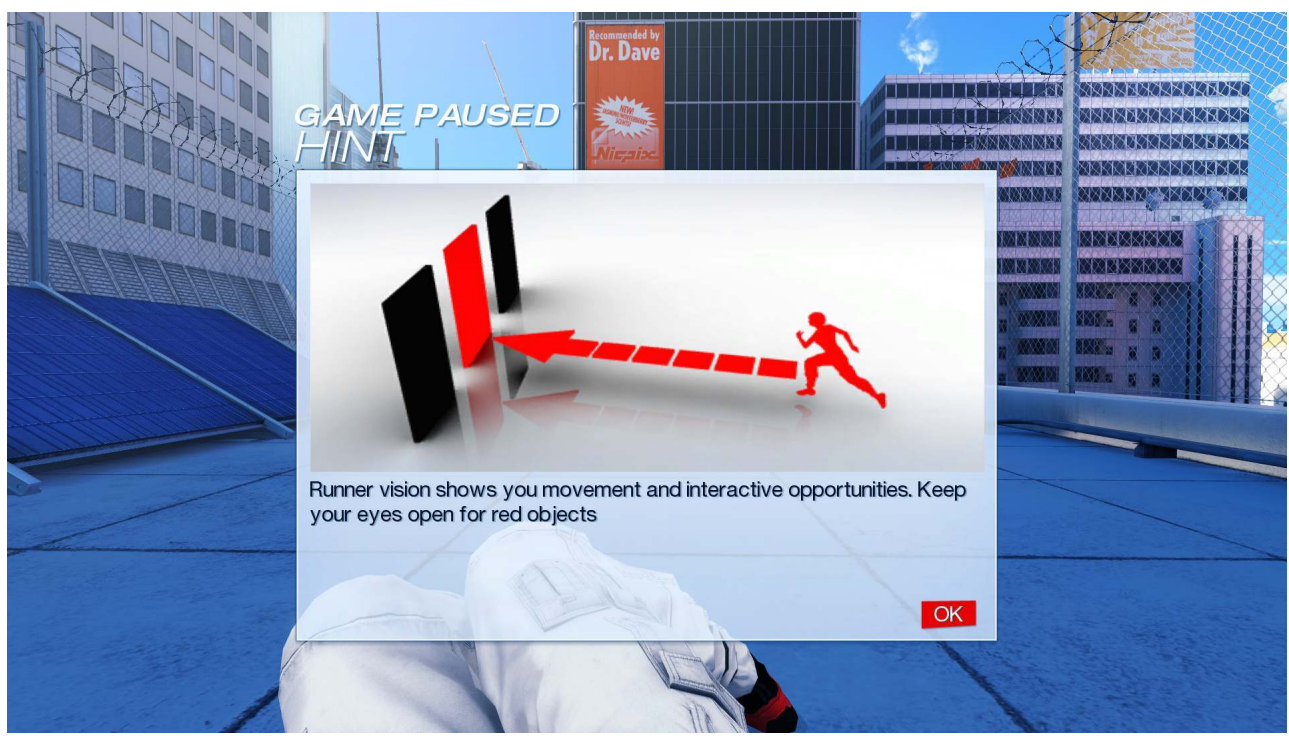

Écran du tutoriel de Mirror's Edge (EA DICE, 2008) introduisant le « Sens urbain ».

Face à ces différentes possibilités d'attribution du foyer de la perception, on constate qu'Allison aborde exclusivement les éléments de focalisation dans une perspective narrative sans tenir compte de leur statut ludique en tant que système de guidage spationarratif destiné au joueur. En interprétant le «Sens urbain» dans l'optique du personnage, le théoricien lie ce savoir à la dimension narrative : c'est Faith qui sait et qui voit de la sorte. En contrepartie, il néglige la fonction purement vidéoludique de ces éléments. Ceux-ci véhiculent bien, et peut-être d'abord, des informations nécessaires à la jouabilité beaucoup plus qu'à l'histoire du personnage. En reprenant les termes de Thabet, ces éléments nous renseignent beaucoup plus sur le joueur que sur la protagoniste. En effet, la décision de désactiver le «Sens urbain » est un choix de joueurexpert et non pas de joueur-novice qui a quant à lui davantage besoin de se faire guider. Or, pourquoi un joueur-expert s'approchant davantage du niveau d'habileté de Faith ne verrait-il plus le surlignage rouge puisqu'il s'agit précisément, selon Allison, d'une 
marque d'expertise ? Dans cette situation, un paradoxe se pose : le tracé rouge est justifié par les compétences spécialisées de Faith, là où pour le joueur, c'est l'absence du tracé rouge qui témoigne de son haut niveau d'expérience. Il serait plus cohérent que le code de couleur se dévoile graduellement au fil de ses innombrables essais-erreurs, notamment parce que les connaissances savantes du joueur devraient l'amener à lire l'environnement comme l'experte Faith.

Ce passage par Allison montre qu'appliquer à la lettre la théorie de Genette fait à tout prendre entrave à une théorisation qui prend aussi en compte la spécificité de la focalisation dans le jeu vidéo. Comme pour Allison, Jonne Arjoranta (2015) priorise dans "Narrative Tools for Games: Focalization, Granularity, and the Mode of Narration in Games » les effets de production de sens narratif au dépend des informations de nature ludique. Les types genettiens de la focalisation sont à nouveau récupérés pour différencier les degrés d'identification au(x) personnage(s). Pour Arjoranta, la vue isométrique des jeux de stratégie en temps réel qui permet au joueur de scruter la région de son choix est en focalisation zéro. Cependant, le «brouillard de la guerre » découle selon lui partiellement de la perception des troupes sur le terrain et cette focalisation devient externe ${ }^{4}$; les informations ici transmises au joueur-commandant s'expliquent en termes diégétiques par l'utilisation des technologies de la communication ou encore par l'usage de la magie par lesdites troupes. Arjoranta $(2015$, p. 6) souligne ainsi que «la focalisation externe est typique des jeux vidéo : l'histoire est racontée de la perspective du protagoniste central, mais à partir d'un point de vue comportemental, sans accès à la conscience du personnage ». Bien qu'il ne se réfère pas au modèle de Jost, il précise qu'il est concevable d'agir et d'incarner le protagoniste à la première personne (donc en ocularisation interne primaire) sans connaître sa «perspective mentale » (en focalisation externe). Toutefois, son exemplification finit par démontrer qu'il ne semble pas entrevoir qu'il soit possible de vivre l'inverse à la troisième personne et d'être au chevauchement de la position d'acteur et de focalisateur. Effectivement, Arjoranta étudie rapidement les séquences de rêverie de Max Payne (Remedy 2001) pour expliquer que la focalisation externe est cette fois-ci « filtrée par les émotions du personnage ». Mais face aux effets oniriques (distorsions optiques, filtre rouge, taches de sang sur les murs, architecture irréaliste et nombreux recadrages automatisés produisant une tension entre enfermement et égarement), n'est-il vraiment pas plus juste de dire que l'expérience des traumatismes intérieurs se joue en focalisation interne et en ocularisation interne secondaire puisque la subjectivité de l'image est créée par tous ces effets ${ }^{5}$ Arjoranta partage exactement l'opinion de Nitsche $(2005,2008)$ qui propose la même analyse. Trop centrés sur la caméra comme foyer de perception, les deux théoriciens restent insensibles à l'attribution évidente des séquences de rêve à Max Payne. Qui plus est, avec la voix «je» utilisée par le détective conjointement à la construction d'une histoire racontée entièrement sur le mode des retours en arrière, l'attribution du "centre d'intérêt » de cette séquence de jeu entière relève de manière on ne peut plus claire de la subjectivité de Max : à la fois comme narrateur, acteur et focalisateur. ${ }^{6}$

Jamais Arjoranta n'explicite les aboutissants vidéoludiques de ses analyses. Cette absence est aussi saillante lorsqu'il examine la «Kill Cam» dans le jeu de tir à la première personne Call of Duty: World at War (Treyarch, 2008). Il souligne que le basculement dans le regard de l'ennemi ainsi que la visualisation de la mort du personnage-joueur depuis son extérieur génèrent des effets de dissociation, d'impuissance et de détresse. De façon surprenante, l'auteur limite ses conclusions à l'idée que ce « changement de perspective 
de dernière minute rappelle [au joueur] que le personnage en est un parmi d'autres, jetable aussitôt qu'il devient inutilisable »(Arjoranta, 2015, p. 11). Arjoranta passe ainsi complètement à côté de l'importance de la « Kill Cam » et de la reprise vidéo du point de vue de l'ennemi comme mécanique permettant de tirer des leçons de jouabilité telles que réévaluer l'échec, se relocaliser à un poste moins à la vue, anticiper la riposte et prévoir de nouvelles tactiques de jeu. Il s'en tient plutôt à l'idée que le travail de la focalisation à l'œuvre ici sert simplement à créer des effets de désincarnation desquels le joueur "pourrait déduire quelques indices sur ce qui aurait changé la situation s'il avait perçu pendant quelques secondes le monde depuis les yeux de son tueur» (Arjoranta, 2015, p. 11). Encore une fois, la focalisation est abordée majoritairement dans l'optique de la relation des personnages et de leurs regards alors que l'exemple de la « Kill Cam » montre que celle-ci est aussi intimement liée à l'expérience du joueur et à l'élaboration de savoirs qui ne se limitent pas qu'au narratif.

\section{La focalis-action dans le jeu vidéo}

Puisqu'à la base la notion de focalisation réfère à la régulation des savoirs, son application dans le cadre du jeu vidéo ne peut pas se limiter à la seule étude du « discours du récit ». Il demeure évident que nous devons tenir compte de la jouabilité. Nous l'avons noté avec Branigan, en plus d'être expérimentateur, le joueur demeure aussi un acteur/agent qui agit et sur lequel on agit. Il est par conséquent difficile de faire l'économie d'un examen approfondi, non plus des seuls savoirs narratifs audiovisualisés, mais aussi des connaissances qui permettent d'agir et d'aiguiller l'action. Par exemple, le "Sens urbain " de Mirror's Edge peut, tel que l'explique Allison, être pensé dans le sillage de la focalisation et renvoyer à la personnalité de Faith comme experte du parcours. Du reste, ce dispositif de guidage spatio-narratif vise d'abord le joueur puisqu'il désigne la route à emprunter et dit au joueur où aller; de la même manière que les interfaces qui agissent comme goulot d'information guident le champ des interactions. ${ }^{7}$ Ce raisonnement nous conduit alors à proposer une distinction entre deux types de focalisation dans les jeux vidéo narratifs : d'une part, la focalisation à valeur narrative et empruntée à la tradition littéraire et cinématographique - apprendre entre autres qu'un personnage était ou n'était pas un traitre sans que cela n'ait d'incidence sur ce que le joueur a dû faire, comme c'est le cas dans Resident Evil (Capcom, 1996), Half-Life 2 (Valve, 2004) ou Metal Gear Solid 3 : Snake Eater (Konami, 2004) - et d'autre part, ce que nous allons appeler la focalis-action qui concerne la régulation des informations et des savoirs à valeur actionnelle. Nous sommes donc enclins à désigner comme un focalis-acteur, à l'image de l'interacteur [interactor] ou du spect-acteur d'œuvres interactives, l'agent situé à l'intersection des sphères d'agentivité associées par Branigan à l'acteur et au focalisateur. Comme nous l'avons noté, le joueur qui a peur dans un jeu d'épouvante à la troisième personne, ou qui panique lors d'une séquence de combat dans un jeu d'action, se pose en focalis-acteur et l'alterbiographie est davantage la sienne.

Afin de bien comprendre les diverses ramifications de la focalis-action, il nous faut à présent expliciter les «modalités du faire ». En littérature et en cinéma, ces modalités sont de l'ordre de la perception et de la cognition. Le lecteur doit se concentrer sur ce qu'il lit; le spectateur est tenu de porter attention à ce qu'on lui montre; et dans un cas comme dans l'autre, il est fondamental de pouvoir comprendre les informations narratives pour suivre le récit. Il n'en demeure pas moins que ne pas saisir un détail, rater 
un indice ou attribuer au mauvais personnage le foyer de la perception n'empêche pas de poursuivre la lecture ou le visionnement. L'interactivité explicite du jeu vidéo demande quant à elle des efforts non-triviaux de participation, qui sont perceptivo-cognitifs et sensorimoteurs. Suivant le modèle sémiotique de la jouabilité inspiré de A.J. Greimas et proposé par Sébastien Genvo (2009, p. 145), il est nécessaire d'analyser le devoir-faire et le pouvoir-faire au sein des différentes séquences narratives canoniques composant un jeu, de sorte à décrire la façon dont une structure encourage à adopter une attitude ludique ("vouloir-faire") en se fondant sur certains savoir-faire. ${ }^{8}$

Il va de soi que le jeu délimite toujours des devoir-faire puisque le joueur doit effectuer des actions incontournables pour progresser. Cette nécessité s'articule inévitablement autour d'un ensemble restreint de pouvoir-faire dans la mesure où certaines conduites sont possibles ou encouragées alors que d'autres sont proscrites. Suivant Genvo, c'est la structure du jeu qui est responsable de véhiculer ces deux modalités. Celles-ci devraient idéalement orienter le joueur sur un vouloir-faire propre aux règles et à la structure du jeu. Cette volonté fait appel à des habiletés préalables ou à développer à partir desquelles il deviendra possible de répondre aux conditions de réussite. Pour cette raison, Genvo situe cette fois le vouloir-faire et le savoir-faire du côté du joueur. En conséquence, et c'est l'intérêt du modèle, les modalités du faire s'articulent de manière cyclique. Du côté de la structure de jeu, la focalis-action sélectionne, présente et audiovisualise des devoirfaire et des pouvoir-faire alors que du côté du joueur, elle signifie une autorégulation de ses vouloir-faire et de ses savoir-faire.

L'exposé de notre concept exige également de tenir compte des positions. En plus des éléments de mise en scène (cadrage, décor, éclairage, jeu d'acteur, etc.), la focalisation au cinéma se déduit suivant Jost à la fois en fonction de considérations pour le point de vue (oricularisation) et pour le point d'écoute (auricularisation) (Gaudreault et Jost, 1990, p.137). Or, pour penser la focalis-action, nous devons porter notre attention sur une troisième position identifiée par Britta Neitzel dans « Narrativity in Computer Games »: «Le point d'action décrit la position à partir de laquelle l'action peut être effectuée et comment elle le sera » (Neitzel, 2005, p. 238). C'est bien la dimension actionnelle qui est mise de l'avant ici. Au demeurant, Nitsche fait allusion à cette dimension. Il remarque que dès lors qu'il est possible de manipuler la caméra interactive, [la focalisation] aide les joueurs à comprendre toute situation de jeu donnée, à contextualiser l'événement [et] à créer des stratégies pour y remédier et interagir avec lui. De cette façon, le processus de compréhension, de planification et d'action positionne le moment narratif de la focalisation au cœur du jeu, comme un moyen puissant de s'engager dans l'espace de jeu (Nitsche, 2008, p. 154).

Une fois que ce rapprochement est effectué, Nitsche n'en explore pas les aboutissants (car ultimement, comment la focalisation est-elle impliquée dans la compréhension et la planification actionnelle ?). Allison note quant à lui que la focalisation est également apparente «dans les affordances pour l'action que celle-ci donne au joueur... » (Allison, 2015, p. 6). Par contre, la réflexion annonce de nouveau que la focalisation communique des affordances au joueur alors que l'analyse se cantonne plutôt à comprendre comment celles-ci éclairent la focalisation interne attribuable à Faith dans Mirror's Edge. Comme l'interprète Allison, les contrôles du jeu favorisent les mouvements durant les parcours tout en rendant la manipulation d'armes désavantageuse (il n'est pas possible de changer d'armes ou de la recharger et sa possession déclenche des confrontations en plus de ralentir les déplacements de Faith). Dans son raisonnement, cela «concorde avec 
l'expérience du personnage dont les buts et les motivations n'impliquent pas de violence » (Allison, 2015, p. 12) et s'aligne sur le registre de la focalisation interne. Dans ce cas, le devoir-faire et le pouvoir-faire viennent encadrer un vouloir-faire qui donne préséance à l'actualisation d'un vouloir-faire et d'un savoir-faire non-violents ; bien que le joueur puisse tout de même décider d'engager des conflits armés. Notre approche souhaite approfondir ces avenues théoriques qui ne sont jamais réellement empruntées et insister sur le trajet de la focalisation vers l'action, et non pas l'inverse.

Des moments-clés de Resident Evil VII : biohazard (Capcom, 2017) ${ }^{9}$ permettent d'illustrer de fort belle façon la direction conceptuelle que nous prenons ici. Au cours du jeu, le personnage-joueur peut visionner quatre cassettes VHS dont le contenu est jouable. Ce contenu présente des espaces qui seront ensuite réexplorés par le joueur. Au deuxième passage, ce dernier bénéficie désormais des indices que l'on peut concevoir comme des devoir-faire et des pouvoir-faire ayant été audiovisualisés au moment où lesdites cassettes ont été jouées. Le joueur devra ensuite cibler les vouloir-faire et les savoir-faire qui permettront sa progression. Dans la première cassette, c'est en tant que caméraman filmant l'entrée d'une petite équipe de tournage dans une maison soi-disant abandonnée que le joueur se voit montrer l'emplacement d'un levier donnant accès à un passage secret dans l'une des pièces de la maison (savoir). S'il peut toujours, par sa connaissance des modalités d'exploration d'un tel jeu d'action-aventure (savoir-faire), découvrir (pouvoir-faire) le levier sans avoir vu la cassette (et attribuer la révélation de cette cachette à une configuration de la focalis-action), il devra absolument passer par ce passage (devoir-faire) et vouloir l'emprunter (vouloir-faire) pour continuer. Dans la troisième cassette, incarnant à la première personne la victime d'un casse-tête qui mène inévitablement à la mort par le feu, le joueur obtient le code - l'une des informations ludiques les plus canoniques - qui lui permet de résoudre le puzzle lorsqu'il s'y frotte à son tour plus tard. Connaitre le code (savoir) permet au joueur de préciser l'ordre de ses actions afin d'effectuer celle (pouvoir-faire) qui lui donne la chance d'éviter l'incendie et de sortir vivant du lieu (devoir-faire). Alors que les informations sélectionnées, présentées et audiovisualisées dans ces cassettes sont doublement diégétisées par le visionnement jouable, leur fonction première est de divulguer des savoirs, des devoirfaire et des pouvoir-faire essentiels à la réussite du joueur. Ce dernier «joue » lesdites cassettes en sachant que ce sont à la fois des moments de focalisation narrative - puisqu'il en apprend sur de précédentes victimes - et de focalis-action vidéoludique.

\section{Perspective sur le savoir et le concevoir interactionnel}

La distinction entre les attitudes propositionnelles et interactionnelles constitue une autre manière d'expliquer la pertinence de réfléchir à la focalis-action. Jean Châteauvert appelle « attitude propositionnelle » ces « opérations d'un degré second » engendrant une «modalisation du contenu des propositions » $(1996$, p. 69) qui déterminent les conditions d'interprétation des procédés énonciatifs et narratifs attribuables à un narrateur ou à un personnage. En lien avec les attitudes subjectives accentuées auparavant par Bal, les attitudes propositionnelles se traduisent par des assertions telles que : je désire que (p)/, je rêve que (p)/, j'affirme que (p)/, j'hallucine que (p), je raconte que (p)/, je documente que (p)/, etc. C'est pourquoi on peut affirmer suivant Châteauvert que "l'analyse de la focalisation rejoint celle de l'énonciation et se conjugue comme un repérage des différents points de vue et attitudes qui colorent un discours » (Châteauvert, 1996, p. 28). 
Dans l'exemple de Max Payne que donne Arjoranta, l'ensemble des effets optiques dénote une attitude propositionnelle relevant du rêve et de l'intoxication du focalisateur qu'est Max.

Force est de constater que dans un jeu vidéo, la modalisation du contenu des propositions ne se déploie pas uniquement sur le plan narratif, mais aussi et surtout sur le plan de mandats et/ou de conditions de jouabilité. Afin d'étudier les opérations de modalisation de contenus destiné à orienter l'action, Hugo Montembeault a introduit le concept d'attitudes interactionnelles en s'appuyant sur les théories de Châteauvert. Ces attitudes correspondent aux modalités du faire que le joueur est en mesure d'attribuer à des sujets, des objets, des contextes ou encore des configurations formelles de jeu. En ce sens, « [1]a détermination d'une attitude interactionnelle fixe alors les conditions d'interprétation et d'interactions vis-à-vis des éléments énonciatifs» (Montembeault, 2014, p. 84-85). L'élaboration de ces attitudes repose sur la capacité du joueur à induire à partir des éléments de jeu des devoir-faire et des pouvoir-faire ainsi que les vouloir-faire et les savoir-faire qui sont sollicités. La relation entre la focalis-action et les attitudes interactionnelles est alors double. D'une part, parce que les devoir-faire et les pouvoirfaire doivent inévitablement être sélectionnés, présentés et audiovisualisés par le système de jeu. D'autre part, parce que la jouabilité du joueur est toujours le résultat d'une configuration (ou autorégulation) de ses savoir-faire et de son vouloir-faire. Afin d'argumenter ce rapprochement conceptuel, nous allons nous concentrer sur l'étude des Spiderbugs, des insectes rampants mi-araignée et mi-scorpion introduits dans le niveau huit du jeu Metro : Last Light (4A Games, 2013).

Dans le niveau en question, le personnage-joueur incarné à la première personne, Artyom, accompagne un personnage non-joueur, Pavel, qui le guide dans les tunnels abandonnés du réseau souterrain du métro. À un moment, les deux acolytes doivent utiliser un vieil ascenseur dont la cage en grillage métallique laisse voir la périphérie. Durant la montée vers le plancher supérieur, le bruit du mécanisme éveille les Spiderbugs. Agressés par la cacophonie, ceux-ci grimpent sur les parois horizontales de la cage d'ascenseur de sorte que le joueur n'a d'autre choix que de tirer sur leur abdomen (Figure 5). 


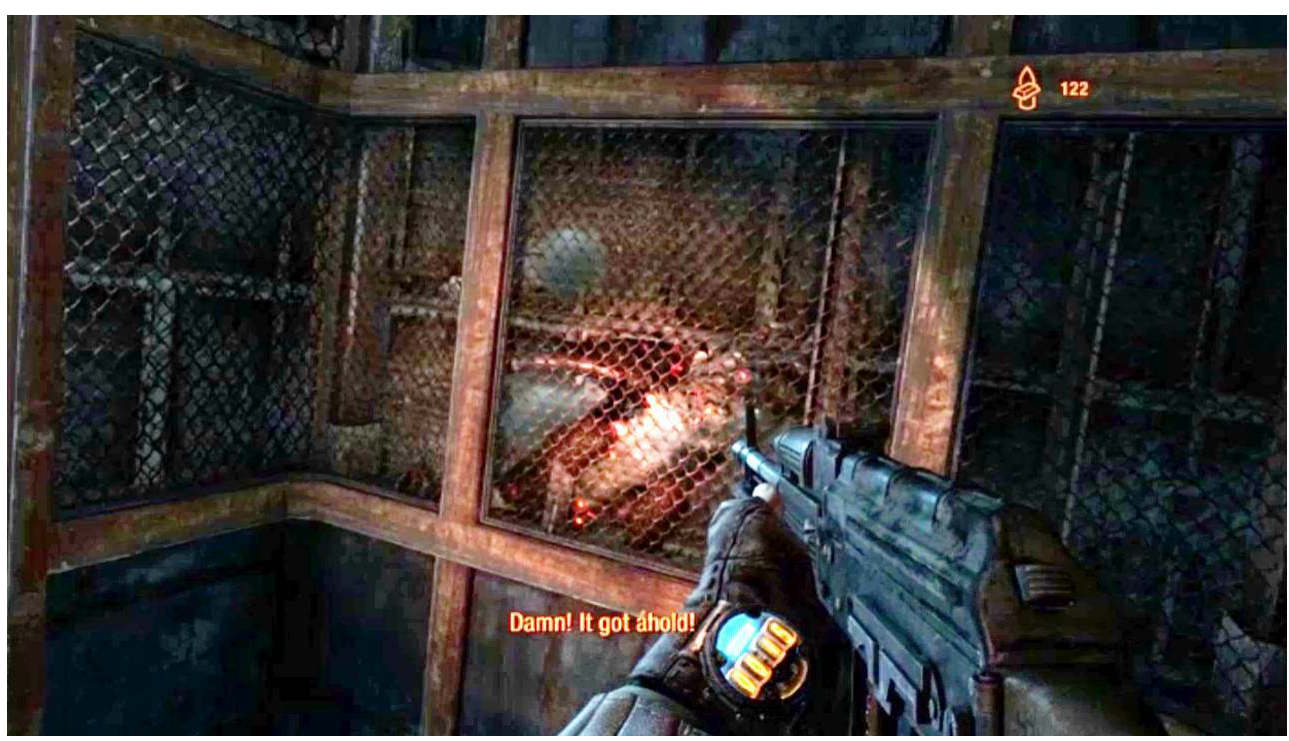

Encagé dans l'ascenseur, le joueur de Metro : Last Light (4A Games, 2013) apprend que c'est dans l'abdomen qu'il doit tirer pour tuer les Spiderbugs.

Après les avoir éliminés, Pavel s'exclame: «Tu vois leur ventre? C'est le seul point vulnérable! Leur armure sur leur dos est trop dure ». On comprend qu'ici, la réplique informative (savoir) s'adresse tout autant au joueur qu'à Artyom. L'ascenseur opère ainsi une restriction du champ actionnel et constitue un dispositif visuo-spatial et sonore. Cette stratégie de design localise les dialogues de Pavel à proximité du point d'écoute, produit le vacarme souhaité (une forme de savoir), régule les déplacements et le champ de vision du personnage (pouvoir-faire) et dirige la mécanique du tir sur l'abdomen des mutants (devoir-faire). Cette configuration de la focalis-action fait directement office de goulot d'information à propos de l'attitude interactionnelle des mutants ; à savoir que ces derniers sont non seulement sensibles aux bruits, mais aussi que leur point faible se situe au niveau de l'abdomen.

Plus loin dans la mission, Artyom et Pavel arrivent devant un couloir bloqué par plusieurs toiles d'araignée. Pavel improvise une torche à l'aide d'un morceau de bois qu'il enflamme avec son briquet. Cette torche lui permet de brûler les toiles d'araignée qui obstruent le passage. En progressant de façon linéaire dans le couloir sombre, des Spiderbugs surgissent sur le chemin des personnages. Dans les premiers affrontements, le joueur n'a qu'à rester auprès de Pavel qui neutralise les mutants à l'aide de sa torche et des toiles d'araignée environnantes qu'il enflamme (pouvoir-faire). Le joueur saisit à cet instant que les Spiderbugs profitent de l'obscurité pour foncer sur leur proie alors que la lumière les effraie (savoir). La disposition architecturale - par endroit en cul-de-sac et par endroit linéaire - ainsi que l'utilisation que fait Pavel du feu et des toiles d'araignée pour éliminer les Spiderbugs forment une configuration de la focalis-action (Figure 6). 
Figure 6

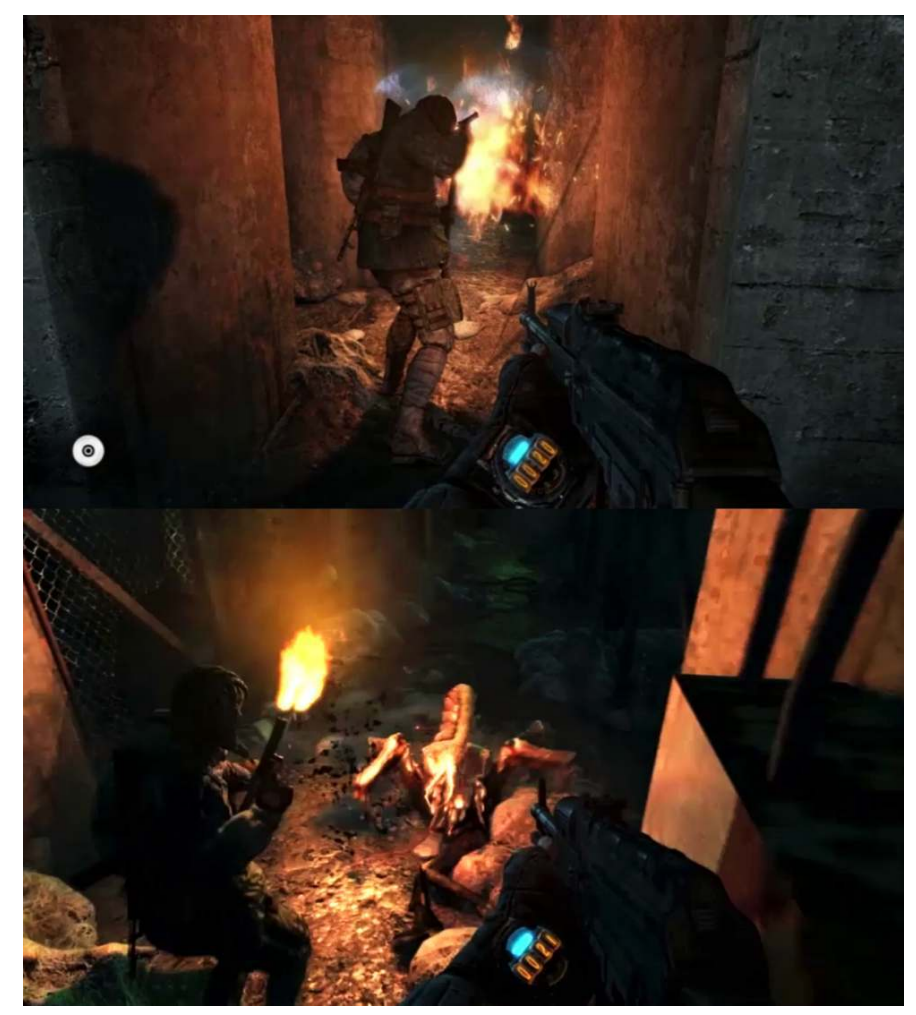

Dans Metro : Last Light (4A Games, 2013), Pavel, le personnage non-joueur, enflamme une toile d'araignée afin de souligner l'importance du feu (image du haut) et repousse un Spiderbugs à l'aide de sa torche (image du bas).

Celle-ci rend intelligible les attitudes interactionnelles associées au briquet, aux toiles d'araignée et aux monstres, permettant au joueur de concevoir les modalités du faire. D'un côté, l'action de Pavel communique des pouvoir-faire à propos du briquet. C'est une source d'éclairage pour s'orienter, mais aussi un outil utilisé soit pour repousser directement les Spiderbugs, soit pour brûler les toiles d'araignée pour accommoder la vision, dégager la voie et incendier les mutants. D'un autre côté, lesdites toiles d'araignée offrent des pouvoir-faire et s'ouvrent à des vouloir-faire. Bien que cela demande plus d'habileté d'exécution (savoir-faire), le joueur peut choisir de brûler les Spiderbugs avec synchronisme afin de propager du feu sur les créatures et d'éviter de gaspiller des munitions.

La focalis-action se veut ainsi une stratégie de design régulant l'information vidéoludique par le biais du narratif permettant au joueur d'élaborer des attitudes interactionnelles. De surcroit, et c'est un aspect fondamental qui a été étonnamment passé sous silence par les études du jeu vidéo, elle est un outil d'analyse pour comprendre comment le joueur, en s'attribuant le rôle de focalis-acteur, régule lui-même ses propres vouloir-faire et savoirfaire. L'articulation de la jouabilité dépend directement de la manière dont, d'une part, le joueur conçoit ses devoir-faire et ses pouvoir-faire et, d'autre part, gère ses savoir-faire et ses vouloir-faire. C'est lui qui est entièrement (parce que sans l'aide de Pavel) responsable de la configuration de l'information visuelle, sonore et actionnelle lorsqu'il affronte seul les mutants. En effet, c'est lui qui décide de brûler les toiles d'araignée pour faciliter sa vision et sa navigation ou encore de les conserver pour neutraliser les Spiderbugs sans dépenser de munitions. C'est au joueur que revient la gestion de son émission sonore en 
choisissant de courir bruyamment, de marcher silencieusement, d'utiliser discrètement son briquet ou encore d'employer son arme à feu; ce qui aura une incidence directe sur la quantité de Spiderbugs à éliminer. C'est bel et bien le focalis-acteur qui détermine comment manipuler l'emplacement de son point d'action et de sa source d'éclairage, soit en fonçant rapidement vers les Spiderbugs pour les éclairer aussitôt avec le briquet, soit en reculant dans l'obscurité en direction d'une toile d'araignée pour amener les créatures à foncer vers le personnage-joueur juste avant de mettre le feu à ladite toile.

La fonction même des tutoriels narrativisés (au début ou au cours du jeu) est de toute évidence de l'ordre de la focalis-action puisque la présentation des savoirs est dirigée vers les devoir-faire et les pouvoir-faire. De même, l'intérêt de consulter une soluce ( walkthrough) écrite ou filmée (par un Let's player par exemple) de Metro: Last Light repose sur le repérage des configurations de la focalis-action attribuables au joueur-expert comme focalis-acteur. Un joueur-novice pourra alors mieux saisir la manière d'éliminer les Spiderbugs. Ne disposant pas des compétences nécessaires pour remarquer les configurations de la focalis-action orchestrées par le design de jeu et concevoir les attitudes interactionnelles qui en découlent, ce dernier se tourne en fait vers les récits alterbiographiques de joueurs-experts.

\section{Le pouvoir intrinsèquement ludique de la focalis- action}

Si, pour Gardies (1988), la focalisation au cinéma confère un pouvoir ludique à l'énonciateur (et, préciserons-nous, au réalisateur ou au cinéma lui-même), il convient alors de conclure en insistant sur le pouvoir à l'évidence frontalement ludique de la focalis-action. Ce pouvoir étant tenu d'assurer un équilibre entre l'intelligibilité et la difficulté des échanges ergodiques entre le jeu et le joueur, nous devons souligner - en suivant la réflexion de Gardies - que la focalis-action repose à la fois sur une relation de confrontation et de coopération. Qu'il soit articulé pour faciliter ou complexifier l'action, le jeu se déploie autour de la régulation des modalités du faire. Sur le plan du design, cela concerne l'intégration et la communication des devoir-faire et des pouvoir-faire par l'entremise du narratif. Du point de vue du joueur, ce pouvoir se conçoit plutôt dans la régulation de ses vouloir-faire et de ses savoir-faire qui détermine comment son activité perceptivo-cognitive et sensorimotrice sera exécutée. Cette dimension personnalisée et personnalisable de la jouabilité renvoie au foyer des configurations de la focalis-action qui est attribué au joueur comme focalis-acteur. L'expérience vidéoludique est en fait toujours filtrée par l'attitude subjective du joueur compris comme " centre d'intérêt » et non pas uniquement par le design de jeu, la caméra virtuelle ou les personnages.

L'étude du pouvoir ludique de la focalis-action à travers les stratégies de design et les stratégies de jouabilité indique que celle-ci est une affaire de restriction ludonarrative et actionnelle qui ne relève pas simplement du jeu, mais aussi et surtout du joueur. En révélant l'activité d'attribution des savoirs et de configuration des faires du joueur comme focalis-acteur, nous avons souhaité expliciter la relation bidirectionnelle entre l'action et la focalisation. Cette ouverture conceptuelle pose les jalons d'une théorie de la focalisa(c)tion plus affranchie de ses origines littéraires et cinématographiques, tout en étant en mesure d'examiner cette part de l'expérience reposant sur une production 
émergente et procédurale de l'information et de l'action vidéoludiques. Il y a toujours du jeu ainsi qu'une dimension aléatoire dans le jeu.

\section{BIBLIOGRAPHIE}

ALLISON, F. (2015), « Whose mind is the signal? Focalization in video game narratives », in Digital Games Research Association (DiGRA), Acte du Colloque : «Diversity of Play », Leuphana University (Lüneburg, 14-17 May 2015), http://www.digra.org/digital-library/publications/whose-mind-isthe-signal-focalization-in-video-game-narratives/

ARJORANTA, J. (2015), « Narrative Tools for Games: Focalization, Granularity, and the Mode of Narration in Games », Game and Culture, 12 (7-8) pp. 696-717.

ARSENAULT, D., CÔTÉ, P. \& LAROCHELLE, A. (2015). « The Game FAVR : A Framework for the Analysis of Visual Representation in Video Games ", Loading... The Journal of the Canadian Game Studies Association, 9,(14), pp. 88-123, http://journals.sfu.ca/loading/index.php/loading/article/ view/155/190

BAL, M. (1977), « Narration et focalisation. Pour une théorie des instances du récit », Poétique, $\mathrm{n}$ - 29 , pp. 107-127

BRANIGAN, E. (1992), Narrative Comprehension and Film, Londres: Routledge

CALLEJA, G. (2009), « Experiential narrative in game environments », Digital Games Research Association (DiGRA), Actes du colloque : «Breaking New Ground: Innovation in Games, Play, Practice and Theory» (Londres, septembre 2009), http://www.digra.org/digital-library/publications/ experiential-narrative-in-game-environments/

CALLEJA, G. (2011), In-Game: from immersion to incorporation, Cambridge, MA: The MIT Press.

CHÂTEAUVERT, J. (1996), Des mots à l'image. La voix over au cinéma, Paris : Méridiens Klincksieck.

GARDIES, A. (1988), « Le pouvoir ludique de la focalisation », Protée, « Le point de vue fait signe », 16 (1-2), pp. 139-145.

GAUDREAULT, A. et JOST, F. 1990. Le récit cinématographique, Paris : Nathan.

GENETTE, G. (1972), Figures III, Paris : Éditions du Seuil.

GENETTE, G. (1983), Nouveau discours du Récit, Paris : Éditions du Seuil.

GENVO, S. (2009), « Understanding Digital Playability », in B. Perron et M.J.P. Wolf (dir.), The Video Game Theory Reader 2, New York: Routledge, pp. 133-149.

GENVO, S. (2008), «Caractériser l'expérience du jeu à son ère numérique : pour une étude du "play design" ", Acte du Colloque : « Le jeu vidéo : expériences et pratiques sociales multidimensionnelles », ACFAS 2008, http://www.ludologique.com/wordpress/?p=105

JOST, F. (1987), L'Eil-caméra : entre film et roman, Paris : Presses Universitaires de Lyon.

LAGNY, M., ROPARS-WUILLEUMIER, M.-C. \& SORLIN, P. (1984), « Le récit saisi par le film » in HorsCadre, «Cinénarrable », $\mathrm{n}^{\circ} 2$, pp. 99-123. 
METZ, C. (1991), L'énonciation impersonnelle, ou le site du film, Paris : Méridiens Klincksieck.

MONTEMBEAULT, H. (2014), De la réception filmique à la participation ludique. Prolégomènes d'une études sur l'énonciation vidéoludique, mémoire de maîtrise, Université de Montréal.

NEITZEL, B. (2005), « Narrativity in Computer Games », in J. Raessens et J. Goldstein (dir.), Handbook of Computer Games Studies, Cambridge, MA: The MIT Press, pp. 227-245.

NITSCHE, M. (2005), « Focalization in 3D Video Games », in Conference Proceedings: Future Play, Lansing, Michigan (13-15 octobre), http://dm.lcc.gatech.edu/ nitsche/download/ Nitsche_Focalization_05.pdf

NITSCHE, M. (2008), Video Game Spaces: Image, Play and Structure in 3D Worlds, Cambridge, MA: The MIT Press.

PERRON, B. (1991), Dans le cadre de la focalisation : Le point de vue du spectateur, mémoire de maîtrise, Université Sorbonne Nouvelle - Paris 3.

SIMON, J. (1983), «Énonciation et narration. Gnarus, auctor et Protée », Communication, «Énonciation et cinéma », nº 38, pp. 155-191.

THABET, T. (2015), Video Game Narrative and Criticism: Playing the Story, Basingstoke: Palgrave Macmillan.

\section{NOTES}

1. Toutes citations d'origine anglophone utilisées dans ce texte ont été traduites par nous.

2. La figure 1 de l'article de Nitsche (2005) montre même Lakitu derrière le plombier dans le reflet d'un miroir ; par équité pour Nitsche, notons qu'il a retiré ce passage dans son ouvrage (Nitsche, 2008). Nous l'introduisons toutefois dans notre propre figure 2 pour conserver l'exemplification du propos.

3. Pour Genette (1983, p, 48), «il n'y a pas de personnage focalisant ou focalisé : focalisé ne peut s'appliquer qu'au récit lui-même...». En développant cette ligne de pensée sur le plan philosophique, et si jouer c'est toujours être joué (Gadamer), nous pouvons affirmer que le joueur demeure toujours le focalisé du jeu dans la mesure où le système ne cesse de suivre, de mesurer et de s'adapter à ses actions et à ses décisions. D'un autre côté, le joueur est aussi toujours en train de focaliser le jeu (son objet de la focalisation) par sa jouabilité. De nouveau, la distinction entre focalisant ou focalisé demeure un fait d'attribution. Au bout du compte, c'est littéralement une question de perception.

4. Puisqu'il ne nomme pas en toutes lettres ce type, nous le déduisons de son argumentation.

5. Et si l'on appliquait le cadre d'analyse proposé par Arsenault, Côté et Larochelle (2015), nommé le «Game FAVR » (Framework for the Analysis of Visual Representation in Video Games), il pourrait s'agir soit d'ocularisation externe zéro ergodique (soit un point de vue transparent qui favorise la jouabilité), soit d'ocularisation externe joueur tangible (une médiation visuelle dont les effets appuient et/ou intensifient les événements se déroulant dans l'espace de jeu). En fait, les auteurs évoquent la notion de focalisation, mais privilégient celle d'ocularisation afin de mieux caractériser les divers régimes graphiques du jeu vidéo et adaptent par le fait même le concept de Jost en proposant d'autres types (p. 105-109). Pour étudier l'intelligibilité des dimensions ergodiques ou interactives de l'image, les auteurs s'intéressent donc au rôle de la médiation visuelle, et non pas de la fiction, (p. 93). Mais dès lors que l'on considère l'importance de ladite fiction vis-à-vis la transmission et l'intelligibilité des modalités du faire vidéoludique, force est de constater que le concept de focalisation reste incontournable. En effet, la narration interactive véhicule couramment (de manière plus ou moins ostentatoire) des savoirs nécessaires 
à la réalisation des objectifs et, comme nous l'avons montré, il paraît difficile d'évacuer le rôle du joueur dans l'attribution du foyer de la perception.

6. Nitsche $(2005,2008)$ développe aussi cet argument contestable lorsqu'il s'intéresse à Prince of Persia: The Sands of Time (Ubisoft, 2003).

7. Proposés par le design et parfois reconfigurables par le joueur, les interfaces relèvent toujours d'une régulation du savoir et du faire, d'où leur relation directe avec ce que nous allons nommer la focalis-action. Qu'elles soient matérielles (contrôleur, haut-parleur, écran, etc.), ou logicielles (jauge de vie, nombre de munitions, carte ou radar interactif, inventaire, etc.), diégétisées ou non-diégétiques, les interfaces sont toujours le site d'une sélection, d'une présentation et d'une audiovisualisation de l'information ludonarrative.

8. Les traductions sont tirées d'un acte de colloque non-publié mis en ligne par Genvo (2008), mais nous renvoyons à la pagination de la version publiée (2009) en anglais qui comporte des petites reformulations.

9. Contrairement aux principaux jeux de la franchise qui se joue à la troisième personne, ce septième opus se déroule entièrement à la première personne (qui plus est en réalité virtuelle via la PlayStation VR).

\section{RÉSUMÉS}

Cet essai porte sur l'un des concepts phares de la narratologie, à savoir la focalisation. Après un survol des théories littéraires et cinématographiques, en commençant évidemment avec l'introduction de la notion par Gérard Genette, il s'agira d'exposer son utilisation en études du jeu vidéo et de souligner les faiblesses ainsi que les contresens d'une application trop ancrée dans une perspective narrative. C'est en mettant l'accent sur l'expérience du joueur, en considérant non seulement le point de vue et le point d'écoute mais aussi le point d'action, et en tenant compte des «modalités du faire » qui encadrent et induisent la jouabilité, qu'une importante nuance sera proposée. En effet, il demeure tout aussi fondamental de s'intéresser à la régulation des informations diégétiques à valeur actionnelle par le design de jeu qu'à la manière dont le joueur lui-même utilise ses savoir-faire. Ces phénomènes qui seront définis en termes de focalisaction.

This essay deals with one of the key concepts in narratology, namely the focalization. It will present a quick overview of the theories in literary and film studies, starting with Gerard Genette who first introduced the notion. It shows how it was used in video game studies in order to underline the weaknesses and narrow interpretations of an application too strongly rooted into a narrative approach. Nuances are then proposed through an emphasis on the player experience, a consideration not only for the point of view and the point of listening, but also for the point of action and, finally, an account of the "modalities of doing » that frame and guide gameplay. In fact, it is fundamental to question the regulation of diegetic information with an action value by the game design, alongside how the player himself uses his own knowledge and know-how. Those phenomenons will be defined in terms of focaliz-action. 
INDEX

Keywords : focalization, narratology, gameplay, video game, film, literature, knowledge, knowhow

Mots-clés : focalisation, narratologie, jouabilité, jeu vidéo, film, littérature, savoir, savoir-faire

\section{AUTEURS}

HUGO MONTEMBEAULT

Université de Montréal

\section{BERNARD PERRON}

Université de Montréal 